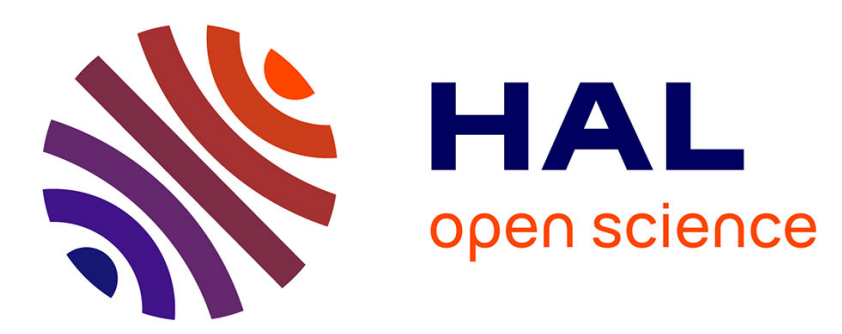

\title{
Probing multilayer stack reflectors by low coherence interferometry in extreme ultraviolet
}

Sébastien de Rossi, Denis Joyeux, Pierre Chavel, Nelson de Oliveira, Marieke Richard, Jean-Yves Robic

\section{- To cite this version:}

Sébastien de Rossi, Denis Joyeux, Pierre Chavel, Nelson de Oliveira, Marieke Richard, et al.. Probing multilayer stack reflectors by low coherence interferometry in extreme ultraviolet. Applied optics, 2008, 47 (12), pp.2109. hal-00557990

\section{HAL Id: hal-00557990 \\ https://hal-iogs.archives-ouvertes.fr/hal-00557990}

Submitted on 15 Feb 2012

HAL is a multi-disciplinary open access archive for the deposit and dissemination of scientific research documents, whether they are published or not. The documents may come from teaching and research institutions in France or abroad, or from public or private research centers.
L'archive ouverte pluridisciplinaire HAL, est destinée au dépôt et à la diffusion de documents scientifiques de niveau recherche, publiés ou non, émanant des établissements d'enseignement et de recherche français ou étrangers, des laboratoires publics ou privés. 


\title{
Probing multilayer stack reflectors by low coherence interferometry in extreme ultraviolet
}

\author{
Sébastien de Rossi,,$^{1, *}$ Denis Joyeux, ${ }^{1}$ Pierre Chavel, ${ }^{1}$ Nelson de Oliveira, ${ }^{2}$ \\ Marieke Richard, ${ }^{3}$ Christophe Constancias, ${ }^{3}$ and Jean-Yves Robic ${ }^{3}$ \\ ${ }^{1}$ Laboratoire Charles Fabry de l'Institut d'Optique, CNRS, Université Paris Sud, \\ Campus Polytechnique, RD 128, 91127 Palaiseau, France \\ ${ }^{2}$ Synchrotron Soleil, L'Orme des Merisiers Saint-Aubin-BP48, 91192 Gif-sur-Yvette, France \\ ${ }^{3}$ CEA/LETI-MINATEC, 17 rue des Martyrs, 38054 Grenoble Cedex 9, France \\ ${ }^{\star}$ Corresponding author: sebastien.derossi@insitutoptique.fr
}

Received 26 November 2007; revised 1 March 2008; accepted 25 March 2008; posted 28 March 2008 (Doc. ID 90043); published 17 April 2008

\begin{abstract}
We use low coherence interferometry to investigate the depth structure of a complex multilayer stack reflector. The probing instrument is an interferometer based on a Fresnel's bi-mirror illuminated by relatively wide-band synchrotron undulator light near $13.5 \mathrm{~nm}$. Simulations clearly confirm that our test object generates two back propagated signals that behave as if reflected on two effective planes. First results in this spectral range may open the way to a new physical approach to extreme ultraviolet sample characterization in the form of line-scan optical coherence tomography. (C) 2008 Optical Society of America

OCIS codes: $\quad 260.3160,260.7200$.
\end{abstract}

\section{Introduction}

EUV optical components are evidently of great interest for the scientific community because of the development of new EUV sources. In the EUV domain, multilayer structures are the only possibility to make near normal incidence mirrors [1]. In the last ten years, great improvements have been achieved in the optical performances of such multilayer mirrors [2] and new optical components [3]. One of those is the development of phase-shift masks (PSMs) for EUV lithography, which will be the component considered in the present article. In the context of the EUV lithography roadmap, the development of PSM [4] is a solution for the phase-enhanced imaging of lithographic masks at $13.5 \mathrm{~nm}$ [5]. For EUV lithography purposes, the designers wish to introduce a phaseshift equal to $\pi$ between the "black" and the "white"

0003-6935/08/122109-07\$15.00/0

(C) 2008 Optical Society of America parts of the mask, so as to enhance the edge steepness in the image of the mask on the wafer. Those novel masks need to be tested at the operation wavelength. To that end, interferometry is the best choice, being the only method which provides direct access to phase. Suitable interferometric systems for EUV have already been demonstrated in various contexts such as the determination of optical constants of materials $[\underline{6}, 7]$, the probing of laser plasma [ㅇ,9], or the metrology at wavelength [10].

Here we bring to light the behavior of a PSM by low coherence interferometric reflectometry. The sample depth structure generates interferograms with contrast modulation, which is the same principle used in optical coherence tomography (OCT). Below, we first explain the experimental context and then show the interferograms. Simulations support our understanding of the interferogram modulation in relation to the resonance structure of multilayer stacks. Concluding remarks extend the idea to possible future work. 


\section{Experimental Configuration}

We first observed the phenomenon during a series of interferometry experiments for phase metrology on PSM components at the XIL beamline of the Swiss Light Source synchrotron [11]. Before describing this first observation in the practical context of the short wavelength spectral range (EUV, soft X rays, etc.), it is appropriate to describe our experimental techniques. To perform EUV interferometry, three specific facts have to be accounted for: 1) spatial coherence requirements imply access to a high luminance. At the moment, synchrotron sources are indeed the most practical and efficient solution, especially when the wavelength must be tuned during a series of tests; 2 ) the complex index shows a refractive index generally close to unity and a fairly large specific absorption; 3) surface smoothness requirements, which scale with wavelength, are quite demanding. The consequence of 2 ) and 3 ) is that classical beamsplitters are very difficult to manufacture (in some cases impossible), are usually chromatic, and show a relatively low efficiency. Although various interferometric systems based on an amplitude division were performed [12-15], our goal was to design a general purpose instrument, i.e., weakly chromatic, having the accuracy required to produce tens of fringes, with a spacing in the range of micrometers to tens of micrometers. In addition, it was specified that it should work close to the zero path difference, in order to accommodate the broadest possible spectral band. We overcame these difficulties by implementing a wavefront division interferometer, namely the Fresnel's bi-mirror under grazing incidence [16]. The system is based on a single block bi-mirror, adjusted by construction close to the null optical path difference (OPD). The angle between mirrors is $0.87 \mathrm{mrad}$. The spatially coherent beam from the output slit of the beamline monochromator situated at approximately $10 \mathrm{~m}$ upstream illuminates first the bi-mirror across the common edge of the two component mirrors, under $3^{\circ}$ grazing. The wavefront is thus divided into two halves that are slightly tilted with respect to each other $(1.74 \mathrm{mrad})$ in order to overlap upon propagation, as is classical with Fresnel's bimirrors. A short distance after reflection $(30 \mathrm{~mm})$, each half beam illuminates the sample on one or several homogeneous zones (neglecting the small overlap produced by propagation); the incidence angle is $6^{\circ}$ from normal. Both half-beams are then reflected toward the detector assembly, $420 \mathrm{~mm}$ downstream. The beam overlap at this place is approximately $730 \mu \mathrm{m}$ (geometrical), and interference can take place. At $13.5 \mathrm{~nm}$ wavelength, the spacing is $7.8 \mu \mathrm{m}$, and the fringe pattern consists of approximately 100 fringes modulated by the Fresnel diffraction of the bi-mirror wedge. The detection system consists of 1 ) in-vacuo conversion of the EUV interferogram into visible light by a grainless phosphor (doped YAG crystal), and 2) in-air imaging of the visible fringe pattern on a CCD camera, which is Peltier cooled for time integration. The fringe field is projected onto the YAG screen at $6^{\circ}$ grazing, thus magnifying the apparent fringe spacing by a factor of 10 compared to the fringe plane spacing. The detected field on the screen is approximately $6 \mathrm{~mm}$ (along fringes) by $10 \times 500 \mu \mathrm{m}$ (perpendicular to fringes).

The way the interferogram is built is illustrated in Fig. 1. To measure the phase-shift produced by a reflecting object with respect to a reference object, it is necessary to simultaneously produce (from the same reflecting substrate) two interferograms: one acting as an reference (zero phase-shift) for the other. To this end, the reflecting object is structured into uniform adjacent regions. One is the reference region (typically a sample with a multilayer stack), the other is the sample region (the complex stack to study). Usually, the reference interferogram is produced when the two half-beams from the interferometer illuminate only the reference regions on the reflector and then interfere. Similarly the sample interferogram is produced when one half-beam illuminates a reference region while the other half-beam illuminates a sample region and then interfere. Of course, it is perfectly possible to arrange the sample and the reference regions so as to produce, as in Fig. 2, a sample interferogram surrounded by the reference interferogram. Such an arrangement should allow for a more accurate determination of the fringe shift. Therefore, the central region with the PSM is surrounded by two reference regions. Each reference region consists of the same Mo-Si multilayer stack with $N$ periods. The PSM sample region, consists of two Mo-Si multilayer stacks with $n$ (superior stack) and $p$ (inferior stack) periods encapsulating a thin layer of silica with variable thickness, and $N=n+p$. The phase-shift produced in the mask is controlled by the silica thickness and by the parameter $n$. The thicknesses of the Mo and Si layers are 2.8 and $4.15 \mathrm{~nm}$, respectively. Materials are deposited by ion beam sputtering on a silicon superpolished substrate. The reflectivity is approximately $60 \%$ at $13.5 \mathrm{~nm}$.

\section{Results}

The XIL beamline of Swiss Light Source was used for these experiments. In Fig. 2(a) an interferogram obtained with a narrow band illumination $(\Delta \lambda / \lambda=0.1 \%)$ shows fringe shifts between the reference pattern and the PSM pattern. This shift is due to the silica spacer inserted in the multilayer, and it can only be modulo $2 \pi$. The interferogram of the same object, obtained under a wide spectral band illumination $(\Delta \lambda / \lambda \approx 6 \%)$ for the reference pattern, shows a wave packet centered on the zero geometrical path difference. For a better representation, Fig. 3(c) plots the local contrast $C$ of the interferogram. It is defined as usual as $C=\left(I_{\max }-I_{\min }\right) /$ $\left(I_{\max }+I_{\min }\right)$ and computed by local Fourier analysis of the interferogram [17].

For the PSM region, the interferogram in Fig. 2(b) shows wave packet splitting into two parts as can be seen in Fig. 3(b) and 3(c). The separation of the two parts depends essentially of the superior stack size $(n)$.

These experimental observations are the consequence of a wide-band illumination of a multieffective 


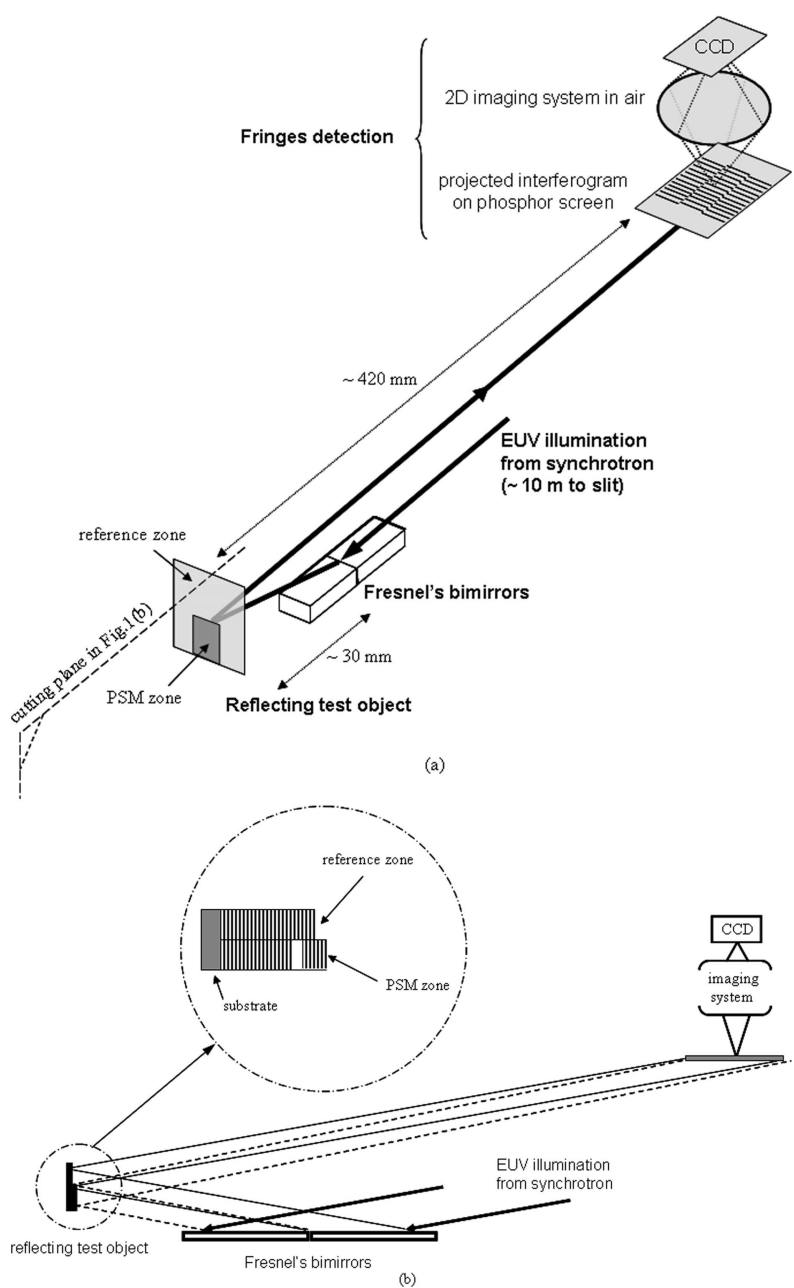

Fig. 1. Phase difference measurement using a Fresnel's bi-mirror interferometer. Through a suitable alignment of zone boundaries relative to the two mirrors common edge, three fringe fields are produced, which are shifted according to the sample characteristics.

plane structure. It is in fact low temporal coherence interferometry. This is the principle of OCT, which is nowadays a widely used non invasive cross-sectional imaging technique in the visible or near-infrared range, especially in a variety of medical fields [18].

While the concept of low coherence interferometry explains the separation between the top and bottom multilayer structures in Fig. 3, at this stage it is nevertheless appropriate to mention the differences between this situation and the usual context of OCT. Typically, OCT uses a Michelson interferometer with a moving mirror in the reference arm. The object arm in OCT contains a biological sample, typically a strongly scattering and weakly absorbing medium composed of layers with diverse scattering coefficients and complex refractive indices. By the fundamental property of the coherence length, for any position of the reference arm, interferences are visible only with reflected signals corresponding to an OPD of less than the coherence length. A source with a wide spectrum in the visible or near-infrared domain can show a coherence length of a few microns. Indeed, submicrometer axial resolution has been reported on samples with a few millimeters penetration depth [19]. In biological samples, the scattering tissues do not usually present ordered structures at the scale of the optical wavelength. Therefore, the reflected signals that originate interference fringes are assumed independent: each set of interference fringe is interpreted as the signature of one discontinuity in the sample. In addition, imaging is provided by $x-y$ scanning. In our case of multilayer EUV reflectors, the medium shows low scattering, strong absorption, with a typical penetration depth less than $1 \mu \mathrm{m}$, and is intentionally ordered-in fact, it is even nearly periodic - at the scale of the EUV wavelength. The return signals from the various multilayers do not only interfere with the reference, but also with each other, creating coherent phenomena such as group velocity dispersion. Therefore the interpretation of our fringes is not as straightforward as in biological OCT. In addition, in our setup, the OCT temporal signal obtained by scanning the moving mirror is replaced by spatial fringes on the detector. The sample structure is assumed to consist of uniform patches so that we effectively interpret the interferograms in terms of a $1 D$ image in the $x$ direction parallel to the fringes, where interferometry provides depth $(z)$ information.

\section{Simulations}

To explore this phenomenon we have simulated the behavior of this structure in a low coherence Fresnel's bi-mirror interferometer taking account the dispersion of the complex indices [20-23]. By a using standard recursive method [24] we calculate the complex reflectivity $R_{\mathrm{REF}}(\lambda)$ of the reference stack and $R_{\mathrm{PSM}}(\lambda)$ the PSM stack in function of the wavelength and by taking into account the roughness in the interface. The normalized interferograms $I_{\mathrm{REF}+\mathrm{REF}}(x)$ and $I_{\mathrm{REF}+\mathrm{PSM}}(x)$ are simply obtained by doing the incoherent sum of the fields weighted by a reflectivity and the spectral density of the source.

$$
\begin{aligned}
I_{\mathrm{REF}+\mathrm{REF}}(x)= & \sum_{\lambda_{\min }}^{\lambda_{\max }} S(\lambda) \times \mid R_{\mathrm{REF}}(\lambda) \times U_{1}(x, \lambda) \\
& +R_{\mathrm{REF}}(\lambda) \times\left. U_{2}(x, \lambda)\right|^{2},
\end{aligned}
$$

$$
\begin{aligned}
I_{\mathrm{REF}+\mathrm{PSM}}(x) & =\sum_{\lambda_{\min }}^{\lambda_{\max }} S(\lambda) \times \mid R_{\mathrm{REF}}(\lambda) \times U_{1}(x, \lambda) \\
& +R_{\mathrm{PSM}}(\lambda) \times\left. U_{2}(x, \lambda) e^{\frac{4 i \pi}{\lambda}\left(e_{\mathrm{PSM}}-e_{\mathrm{REF}}\right)}\right|^{2} .
\end{aligned}
$$

Where $U_{1}$ (resp. $U_{2}$ ) is the amplitude of the reflected field by mirror one (resp. mirror 2 ) of the bi-mirror, $x$ is the spatial coordinate of the interferogram (perpendicular to the fringes), which codes the path difference, and $e_{\mathrm{REF}}$ (resp. $e_{\mathrm{PSM}}$ ) is the physical thickness of the reference stack (resp. the PSM 

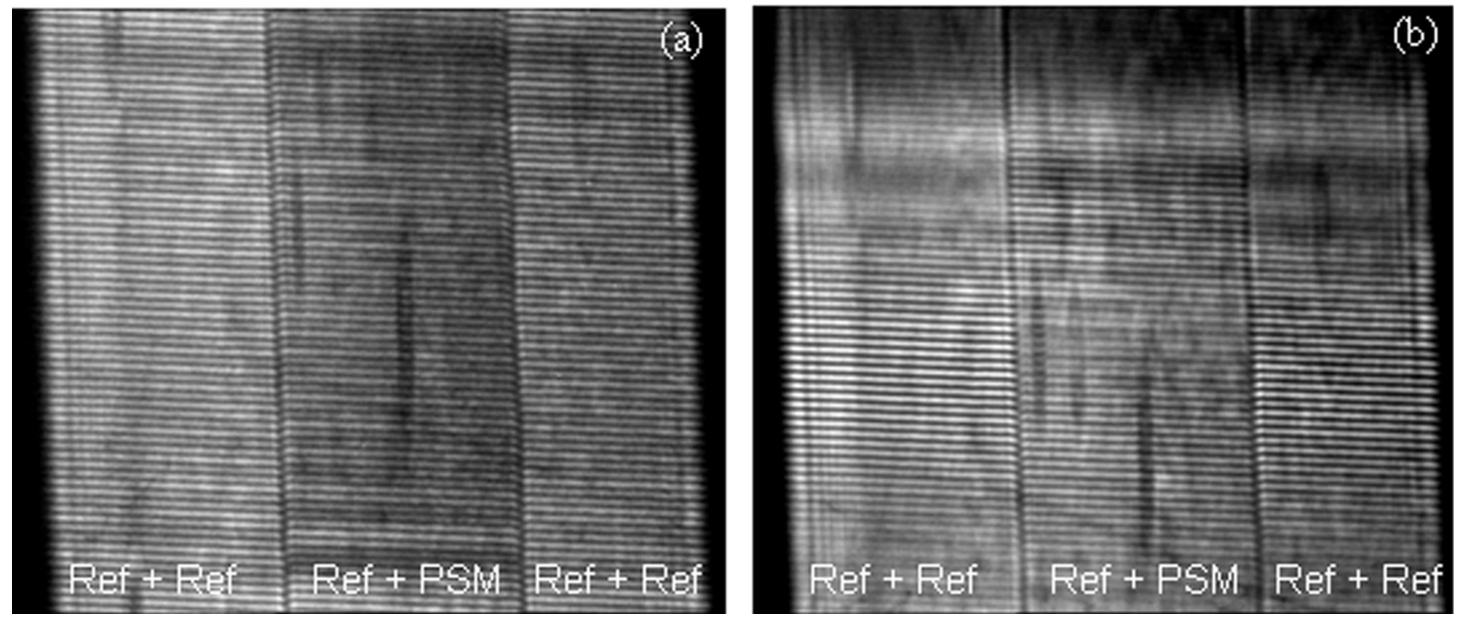

Fig. 2. Interferogram obtained with Fresnel's bi-mirror andPSM component. (a)narrow bandillumination; (b) wide-band illumination: blurring by spectral width is clearly visible. The modulation due to the Fresnel diffraction is also clearly visible in top and in bottom of both interferograms.

stack). $\lambda_{\min }$ and $\lambda_{\max }$ are the boundary of the spectral domain. $S(\lambda)$ is the normalized spectral density of the source that we define as a hypergaussian function of order three with a full width at half maximum at approximately $6 \%$ of the central wavelength $(13.5 \mathrm{~nm})$. Here we take into account the Fresnel diffraction on the edge of the bi-mirror in the expression of the amplitude $U_{1}(x, \lambda)$ and $U_{2}(x, \lambda)$ [25].

Figure 4 depicts the evolution of the contrast versus the delay $(x)$ for different reflective phase structures. The reference region is a Mo-Si stack with 51 periods on a Si substrate. The interference between the two identical reference regions with 51 periods (Fig. 4(a)) gives contrast maximum at the geometrical zero path difference of the interferometer, regardless of the number of periods. When one of the two references is replaced by a multilayer stack with more layers (here 60 periods) the maximum of the contrast (Fig. 4(b)) is shifted ahead (phase advanced). For a multilayer stack with less layers (here 40 periods), the maximum is delayed with respect to the geometrical zero OPD (Fig. $\underline{4(\mathrm{~b})}$ ). As the position of

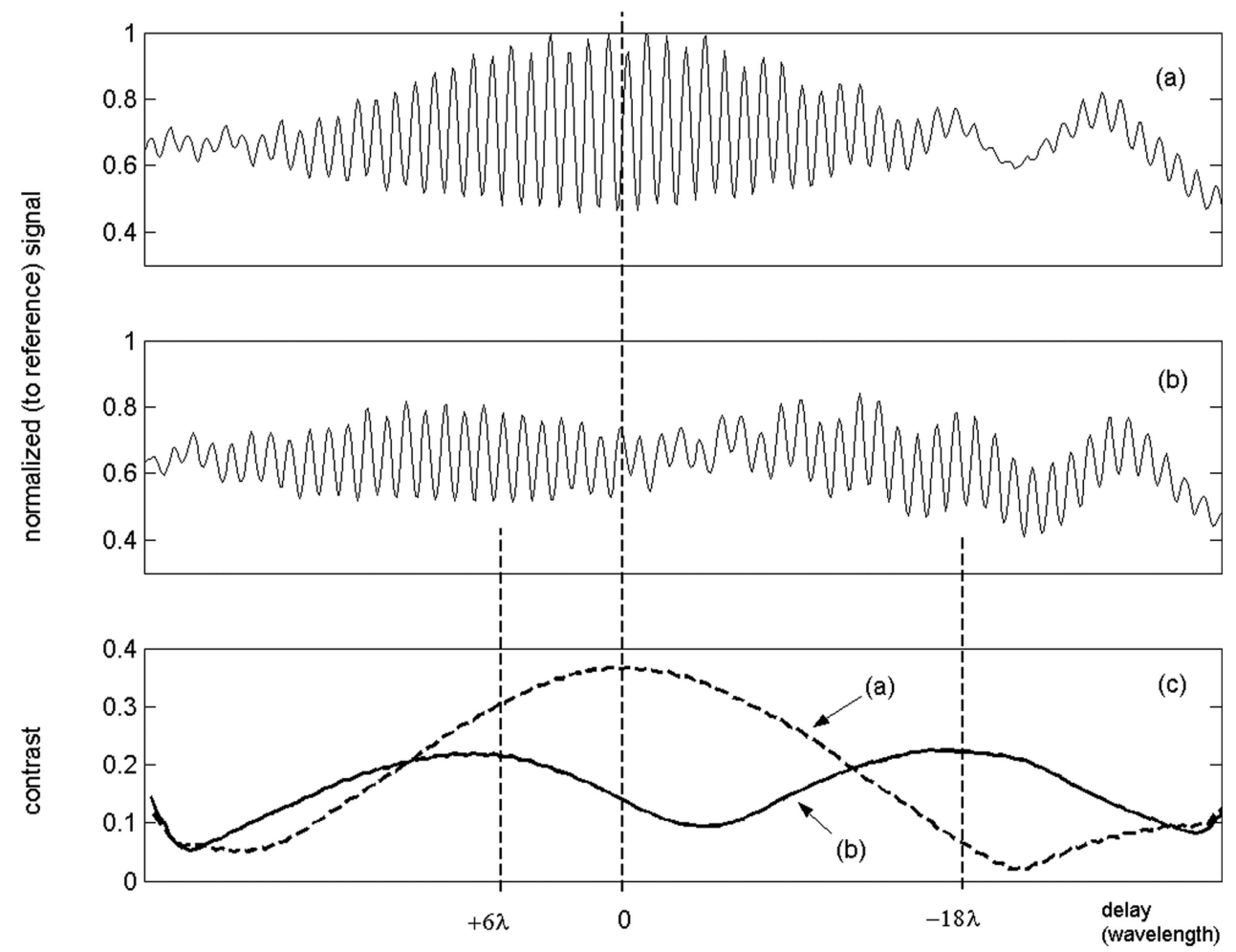

Fig. 3. Profiles (a and b) and contrasts (c) of the experimental interferograms from Fig. 2(b). (a) reference + reference; (b) reference + PSM; (c) contrast plot : reference + reference [dashed curve (a)], reference + PSM [solid curve (b)]. Note : the left here is the bottom in Fig. 2 . 


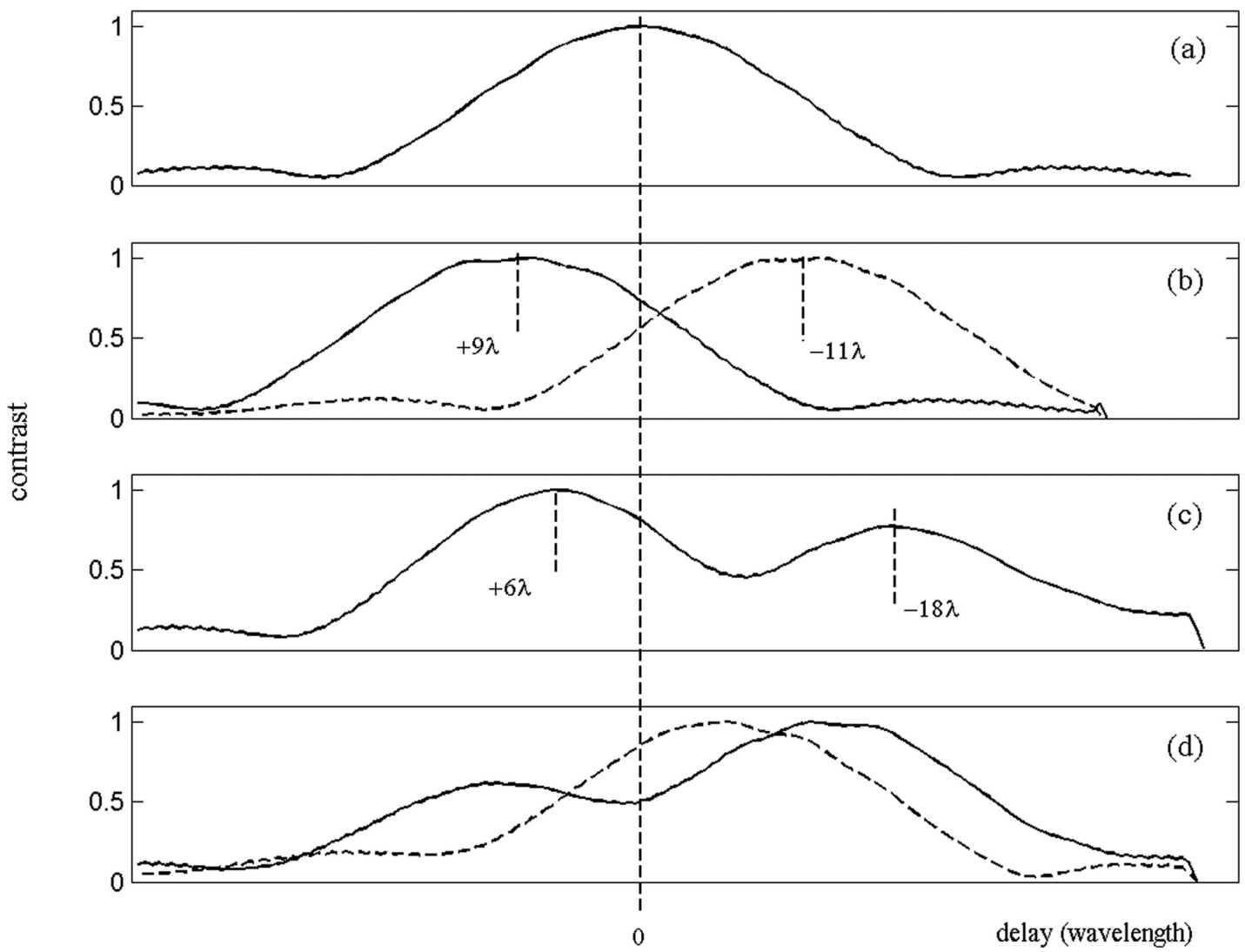

Fig. 4. Contrast of inteferograms simulated with different reflective structures. The reference region is a Mo-Si stack with 51 periods on Si substrate. This region interferes with : (a) reference itself; (b) 60 periods + substrate (solid curve) and 40 periods + substrate (dashed curve); and (c) PSM : 11 periods $+3 \mathrm{~nm} \mathrm{SiO} 2+40$ periods + substrate; (d) PSM with seven periods on the top stack (solid curve) and PSM with three periods on the top stack (dashed curve).

the substrate is the same in the simulations, the shifts are simply due to the geometrical difference of the total stack thicknesses. Now consider the case of PSM component with two substacks (40 at the bottom and 11 on top) separated by a silica spacer. For the approximately $3 \mathrm{~nm}$ thickness of silica, two maxima are observed: one is ahead by 6 fringes; the second one is delayed by 18 fringes (Fig. 4(c)).

To get an intuitive understanding of those effects, the reflection on a multilayer can be considered as a reflection on an effective plane whose depth depends of the number of periods. The depth of the wave packet contrast maximum can be calculated versus the number $m$ of Mo-Si periods in each stack ( $m=n$ on the top stack; $m=p$ on the bottom stack). Taking a perfect and infinitely thin reflector placed on the top of the structure as the reference, the depth of the effective plane is then found to be constant (approximately $10 \lambda)$ for $m>20$. Under 20 periods the depth decreases down to 3.5 fringes for $m=5$. So, for $m=n=11$ on top and $m=n=40$ at the bottom with the spacer in between, we infer that the OPD between wave packets should be approximately $15 \lambda$. In fact we measure $24 \lambda$ (Fig. 3(c))and the simulation with the real structure gives also this same result (Fig. 4(c)).

To understand that fact, we have to consider the resonant effect between the two stacks. The spacer is weakly absorbing. So, we have a Fabry-Perot effect between two multilayer mirrors separated by a $3 \mathrm{~nm}$ silica (Fig. 5). The reflectivity of the top mirror $(n=11)$ is $R_{T}=33 \%$. The reflectivity of the bottom mirror $\left(p=40\right.$ ) is $R_{B}=62 \%$. The resonator finesse with an optical effective thickness $L_{\mathrm{FP}}$ of the cavity, for a central wavelength $\lambda_{0}$ can be written as,

$$
F=\frac{\pi}{a \cos \left[\frac{2 \sqrt{R_{B} R_{T} e^{\beta}}}{1+R_{B} R_{T} e^{\beta}}\right]},
$$

where

$$
\beta=-\frac{2 \pi}{\lambda_{0}} \mathfrak{I}\left(2 L_{\mathrm{FP}}\right)
$$

and

$$
L_{\mathrm{FP}}=N_{\mathrm{FP}}\left(n_{\mathrm{Mo}} e_{\mathrm{Mo}}+n_{\mathrm{Si}} e_{\mathrm{Si}}\right)+n_{\mathrm{SiO} 2} e_{\mathrm{SiO} 2} .
$$

Here $N_{\mathrm{FP}}$ is the number of Mo-Si periods between the effective mirrors (in the stacks) of the Fabry-Perot cavity. $n_{\mathrm{Mo}}, n_{\mathrm{Si}}$, and $n_{\mathrm{SiO} 2}$ (resp. $e_{\mathrm{Mo}}, e_{\mathrm{Si}}$, and $e_{\mathrm{SiO} 2}$ ) are the complex optical indices (resp. the thicknesses) of molybdenum, silicon, and silica layers and $\mathfrak{I}$ designates the imaginary part. For $N_{\mathrm{FP}}=15$ 


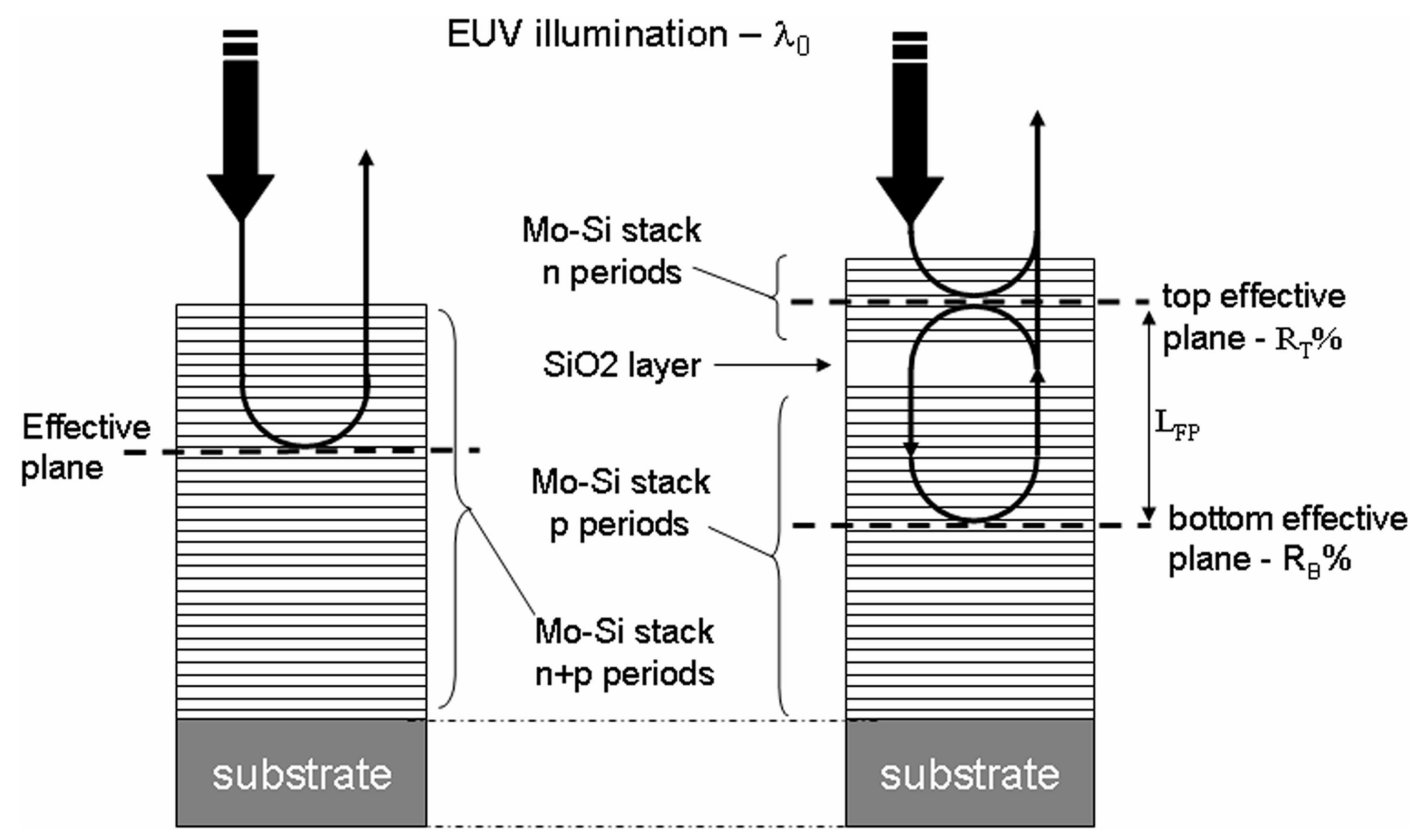

Fig. 5. Sectional scheme of the reference zone (left) and the PSM zone (right). The optical rays describe the reflection of the EUV illumination on the effective planes of the stacks. In the PSM component the effective planes define a Fabry-Perot cavity.

(as mentioned above), we obtain a finesse around 3.5. So, without dispersion, we should have about three or four replica of the wave packets each separated by $15 \lambda$ with decreasing amplitudes. Since the full width at half maximum of the wave packets is approximately $22 \lambda$ (corresponding to a $6 \%$ band spectral illumination filtered by the multilayer), replica overlap, and interfere. Together with the strong dispersion of the mirrors, this effect can account for the observed separation of $24 \lambda$ between the envelope maxima in Figs. 3(c) and $4(\mathrm{c})$. With seven periods in the top stack $(n=7$ and $\overline{p=} 40$ ), the Fabry-Perot effect decreases (Fig. 4(d)). The reflectivity of the first multilayer is smaller. With three periods in the top stack $(n=3, p=40$, and $N=43)$, the effect disappears and the two packets overlap (Fig. 4(d)). We can no longer resolve the resonant effective planes.

\section{Conclusions}

Could the present considerations be developed into a practical OCT technique for the EUV domain, i.e., for probing nanometer scale structures in the limit of their submicrometer penetration depth? Because of the low reflectivity associated with complex refractive indices in that spectral range, high sensitivity detection is needed, and as explained above, the structure periodicity creates group velocity dispersion that must be taken into account in the reconstruction algorithm, be it only by introducing the concept of effective interface planes as we did here. Under these conditions, some modified form OCT may be transferred to the EUV domain, not only for probing multilayer reflectors. One may think, in particular, about plasma diagnostics, where scattering and/or periodicity in the plasma layers within the penetration depth can be investigated.
In conclusion we have shown the first experimental demonstration of a low coherence interferometric reflectometry experiment with a Fabry-Perot resonance in the EUV domain. Theoretical considerations confirm the effect. It could be a new way for probing matter at nanometer scale, possibly with wide-band pulsed sources like high harmonics generation sources.

The authors thank Harun Solak and his colleagues for their invaluable availableness and efficiencies in the optimization of the XIL beamline. This work was supported by the More Moore Integrated Project of the European Union (IST-1-507754-IP).

\section{References}

1. T. W. Barbee Jr., S. Mrowka, and M. C. Hettrick, "Molybdenumsilicon multilayer mirrors for the extreme ultraviolet," Appl. Opt. 24, 883-886 (1985).

2. J. Gautier, F. Delmotte, M. Rouillay, F. Bridou, M. F. Ravet, and A. Jérôme, "Study of normal incidence three component multilayer mirrors in the range $20 \mathrm{~nm}-40 \mathrm{~nm}$," Appl. Opt. 44 (3), 384-390 (2005).

3. R. M. Fechtchenko, A. V. Vinogradova, and D. L. Voronov, "Optical properties of sliced multilayer gratings," Opt. Commun. 210, 179-186 (2002)

4. M. D. Levinson, N. S. Viswanathan, and R. A. Simpson, "Improving resolution in photolithography with a phase-shifting mask," IEEE Trans. Electron Devices ED-29 (1982).

5. T. Ito and S. Okazaki, "Pushing the limits of lithography," Nature (London) 406 (6799), 1027-1031 (2000).

6. J. Svatos, D. Joyeux, D. Phalippou, and F. Polack, "Soft-x-ray interferometer for measuring the refractive index of materials," Opt. Lett. 18 (16), 1367-1369 (1993).

7. C. Chang, E. Anderson, P. Naulleau, E. Gullikson, K. Goldberg, and D. Attwood, "Direct measurement of index of refraction in the extreme-ultraviolet wavelength region with a novel interferometer," Opt. Lett. 27, 1028-1030 (2002).

8. L. B. Da Silva, T. W. Barbee Jr., R. Cauble, P. Celliers, D. Ciarlo, S. Libby, R. A. London, D. Matthews, S. Mrowka, J. C. Moreno, 
D. Ress, J. E. Trebes, A. S. Wan, and F. Weber, “Electron density measurements of high density plasmas using soft x-ray laser interferometry," Phys. Rev. Lett. 74, 3991-3994 (1995).

9. R. F. Smith, J. Dunn, J. Nilsen, J. R. Hunter, V. N. Shlyaptsev, J. J. Rocca, J. Filevich, and M. C. Marconi, "Refraction effects on x-ray and ultraviolet interferometric probing of laserproduced plasmas," J. Opt. Soc. Am. B 20, 254-259 (2003).

10. P.P.Naulleau, K. A. Goldberg, S. H. Lee, C. Chang, D. Attwood, and J. Bokor, "Extreme-ultraviolet phase-shifting point-diffraction interferometer: a wave-front metrology tool with subangstrom reference-wave accuracy," Appl. Opt. 38, 7252-7263 (1999).

11. M. Richard, D. Constancias, D. Joyeux, J. Y. Robic, S. de Rossi, and N. de Oliveira, "EUV phase shift masks samples at wavelength phase shift measurements on dedicated samples," in The Proceedings of the 5th International EUVL Symposium (October 8-10 2006).

12. P. P. Naulleau and K. A. Goldberg, "Dual-domain point diffraction interferometer," Appl. Opt. 38 3523-3533 (1999).

13. J. J. Rocca, C. H. Moreno, M. C. Marconi, and K. Kanizay, "Soft-x-ray laser interferometry of a plasma with a tabletop laser and a Lloyd's mirror," Opt. Lett. 24, 420-422 (1999).

14. L. B. Da Silva, T. W. Barbee Jr., R. Cauble, P. Celliers, D. Ciarlo, J. C. Moreno, S. Mrowka, J. E. Trebes, A. S. Wan, and F. Weber, "Extreme-ultraviolet interferometry at $15.5 \mathrm{~nm}$ using multilayer optics," Appl. Opt. 34, 6389-6392 (1995).

15. Y. Zhu, K. Sugisaki, M. Okada, K. Otaki, Z. Liu, J. Kawakami, M. Ishii, J. Saito, K. Murakami, M. Hasegawa, C. Ouchi, S. Kato, T. Hasegawa, A. Suzuki, H. Yokota, and M. Niibe, "Wavefront measurement interferometry at the operational wavelength of extreme-ultraviolet lithography," Appl. Opt. 46, 6783-6792 (2007).
16. F. Polack, D. Joyeux, J. Svatos, and D. Phalippou, "Applications of wavefront division interferometers in soft x rays," Rev. Sci. Instrum. 66 (2), 2180-2183 (1995).

17. M. Takeda, H. Ina, and S. Kobayashi, "Fourier-transform method of fringe-pattern analysis for computer-based topography and interferogram," J. Opt. Soc. Am. A 72, 156 (1982).

18. D. Huang, E. A. Swanson, C.P.Lin, J.S.Schuman, W. G. Stinson, W. Wang, M. R. Hee, T. Flolte, K. Gregory, C. A. Pulia, and J. Fujimoto, "Optical coherence tomography," Science 254, 1178-1181 (1991).

19. B. Povazay, K. Bizheva, A. Unterhuber, B. Hermann, H. Sattmann, A. F. Fercher, W. Drexler, A. Apolonski, W. J. Wadsworth, J. C. Knight, P. St. J. Russell, M. Vetterlein, and E. Scherzer, "Submicrometer axial resolution optical coherence tomography," Opt. Lett. 27 (20), 1800-1802 (2002).

20. D. L. Windt, "XUV optical constants of single-crystal GaAs and sputtered C, Si, Cr3C2, Mo and W," Appl. Opt. 30, 15-25 (1991).

21. R. Soufli and E. M. Gullikson, "Reflectance measurements on clean surfaces for the determination of optical constants of silicon in the extreme ultraviolet-soft-x-ray region,” Appl. Opt. 36, 5499-5507 (1997).

22. R. Soufli and E. M. Gullikson, "Absolute photoabsorption measurements of molybdenum in the range 60 to $930 \mathrm{eV}$ for optical constant determination," Appl. Opt. 37, 1713-1719 (1998).

23. C. Tarrio, R. N. Watts, T. B. Lucatorto, J. M. Slaughter, and C. M. Falco, "Optical constants of in situ-deposited films of important extreme-ultraviolet multilayer mirror materials," Appl. Opt. 37, 4100-4104 (1998).

24. E. Spiller, Soft X-Ray Optics (SPIE Optical Engineering Press, 1994).

25. M. Born and E. Wolf, Principle of Optics (Pergamon, 1970). 